Sebastian Flinzberg · Rainer Schmelzle

Abteilung für Mund-, Kiefer- und Gesichtschirurgie, Nordwestdeutsche Kieferklinik, Universitätsklinikum Hamburg-Eppendorf

\title{
Rekonstruktion und Stabilisierung im Gesichtsbereich
}

\section{Zusammenfassung}

Traumatische Verletzungen des Gesichtsschädels gehen mit teilweise ausgedehnten Defekten der Gesichtsweichteile und des knöchernen Schädels einher. Die notwendigen Rekonstruktionsverfahren sind in vielen Fälle komplex, schwierig und erfordern einen hohen Zeitaufwand. Moderne Osteosyntheseverfahren und mikrochirurgische Techniken bilden die Grundlage für gute Erfolge und eine zügige Rehabilitation der Verletzten.Die Rekonstruktion des Zahn tragenden Apparats und die exakte Einstellung der Okklusion sind für die Nahrungsaufnahme und die Sprachbildung wichtig.Zur Rekonstruktion bei Defekten stehen vaskuläre und avaskuläre Transplantate zur Verfügung. Mikrovaskuläre Fibula-, Beckenkamm- oder Skapulatransplantate ermöglichen einen kombinierten Weichteil- und Knochenaufbau.Die Weiterentwicklung der dentalen Implantologie ermöglicht in der Folge die Wiederherstellung der Kaufunktion.

\section{Schlüsselwörter}

Mikrochirurgie - Fixateur-interne-Systeme . Osteosynthese - Mittelgesichtsfraktur . Unterkieferfraktur
K schädels und Zatzunges mit teilweise ausgedehnten Verletzungen der Gesichtsweichteile vergesellschaftet und bedürfen einer sorgfältigen Therapie und Rekonstruktion [8]. Gesichtsverletzungen mit späterer Narbenbildung und der häufig folgenden sozialen Stigmatisierung können zu erheblichen psychosozialen Belastungen der Patienten führen. Motilitäts- und Gefühlsstörungen durch Schäden an den Hirnnerven bringen nicht selten Sprachund Schluckveränderungen mit sich. Die Zertrümmerung des Alveolarkamms mit Verlust von Schneide- und Seitenzähnen sowie die dargestellte subtotale Abtrennung der Unterlippe erfordern eine aufwändige Rekonstruktion (Abb. 1).

Die Versorgung der Knochenwunden insbesondere von Frakturen des Unterkiefers und Mittelgesichts ist in der Mund-, Kiefer- und Gesichtschirurgie von zentraler Bedeutung $[3,4]$.

\section{Patienten}

Im Jahr 2000 wurden in der Abteilung für Mund-, Kiefer- und Gesichtschirurgie 397 Frakturen des Gesichtsschädels stationär versorgt.

Bei der Geschlechtsverteilung zeigte sich ein typisches Überwiegen des männlichen Geschlechts $(n=279)$ im Verhältnis zu den Frauen $(n=118)$.

Bei der Altersverteilung der Patienten zeigte sich eine Zunahme der Verletzungsanzahl im Alter von 25-35 Jahren. Die Zahl der Verletzungen fiel dann im Verhältnis zum steigenden Lebensalter ab. Diese Verteilung ist mit der deutlich höheren körperlichen Aktivität der Altersgruppe von 25-35 Jahren zu erklären. $83 \%$ aller Zahnverletzungen fallen jedoch in das Kleinkindes- und Heranwachsendenalter [8]. Bemerkenswerterweise erleiden $50 \%$ aller Kinder vor Abschluss der Schulausbildung in beiden Dentitionen ein oder mehrere Zahntraumen [1]. Hauptursache dieser Verletzungen im Kindesalter sind Stürze. Deshalb sollte auch bei einer unscheinbaren Lippenplatzwunde immer eine genaue intraorale Inspektion durchgeführt werden (Abb. 2).

Aufgrund der mittlerweile hohen Sicherheitsstandards der Fahrzeuge sind durch Verkehrsunfälle mit Aufschlag auf das Lenkrad bedingte Gesichtsschädelfrakturen rückläufig. Im Krankengut der Hamburger Universitätsklinik ist in den letzten Jahren eine Zunahme der Sport- und Freizeitunfälle zu verzeichnen. Bis zu $62 \%$ der Gesichtsschädelverletzungen ereignen sich im Sport und Freizeitbereich, hiervon werden $34 \%$ durch Fahrradstürze und 35\% durch Mannschaftssportarten verursacht. Bis zu 40\% der Verletzen sind alkoholisiert [8]. Diese häufig polytraumatisierten

\footnotetext{
Dr. Dr. Sebastian Flinzberg

Abteilung für Mund-, Kieferund Gesichtschirurgie,

Nordwestdeutsche Kieferklinik, Universitätsklinikum Hamburg-Eppendorf, Martinistraße 52, 20246 Hamburg, E-Mail:mkg@uke.uni-hamburg.de, Tel.:040-428032251, Fax:040-428035467
} 


\section{S. Flinzberg $\cdot$ R. Schmelzle}

\section{Reconstruction and stabilization} in the facial area

\section{Abstract \\ Traumatic injury of the facial skeleton incor- portes defects of the facial soft tissue and of the osseous structures, in some cases exten- sive. The necessary reconstructive measures can prove complex and difficult. Modern methods of osteosynthesis in combination with microsurgical techniques are often the basis for successful and expedient rehabilita- tion of the trauma patients. Reconstruction of the dentoalveolar complex with precise adjustment of the occlusion is important for mastication and phonation. Vascular and avascular transplants are available for recon- struction when there are defects. Microvas- cular fibular, iliac crest or scapular flaps allow simultaneous reconstruction of soft and of osseous tissue. It will be increasingly possible to continue improving effective mastication later with the ongoing developments in the field of dental implantology.}

\section{Keywords}

Microsurgery $\cdot$ Internal fixation systems . Osteosynthesis - Midfacial fractures . Mandibular fractures

Abb. 1> Ausgedehnte Gesichtsverletzung, subtotaler Unterlippenabriss mit Luxation und Verlust von Front- und Seitenzähnen. Mittelgesichtsfraktur auf LeFort-II-Ebene und Nasenbeinzertrümmerung. In diesem Fall wurde eine aufwändige mehrstufige Rekonstruktion notwendig

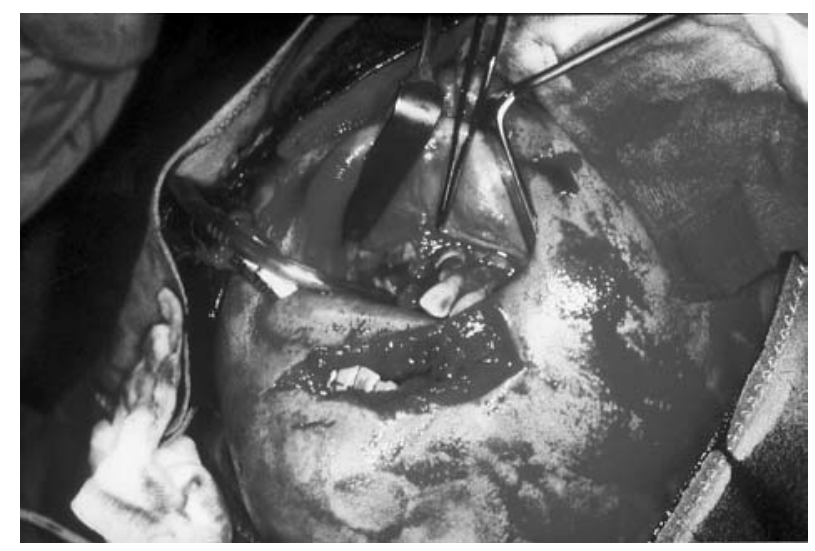

Patienten haben heute dank des hohen Standards der Intensivmedizin, der sicheren Rettungskette und der modernen Entwicklung der unfallchirurgischen Versorgung hohe Überlebenschancen [4]. Eine verbesserte Diagnostik und zügige Überführung in entsprechende Spezialabteilungen sind als Vorteile zu nennen [4].

\section{Therapie}

Bei vorliegenden Knochenverletzungen des Gesichtsschädels stehen die Behandlung und Versorgung von lebensbedrohlichen Blutungen und Schwellungen im Vordergrund, es folgen die möglichst frühzeitige Stabilisierung und Rekonstruktion nach individueller Indikationsstellung.

Die Wiederherstellung der Kontinuität des Unterkieferknochens und der Mittelgesichtsknochen kann an den Ope- rateur und das Osteosynthesematerial hohe Anforderungen stellen, wenn ausgedehnte Trümmerzonen überbrückt und Knochendefekte rekonstruiert werden müssen.

Für die Wiederherstellung der Form und Funktion ist eine exakte Reposition der Gesichtsschädelknochen anzustreben $[3,4]$, bei den unabänderlichen Bewegungen, wie dem Schluckvorgang, müssen die reponierten Knochenstücke zumindest übungsstabil versorgt sein.

In den letzten Jahrzehnten stand u. a. die Miniaturisierung des Osteosynthesematerials im Vordergrund $[2,6,10]$ (Abb. 3).

\section{Frakturversorgung von Mittelgesicht und Unterkiefer}

Die Einteilung der Mittelgesichtsfrakturen erfolgt noch immer auf der Basis der von LeFort 1901 beschriebenen Fraktur-
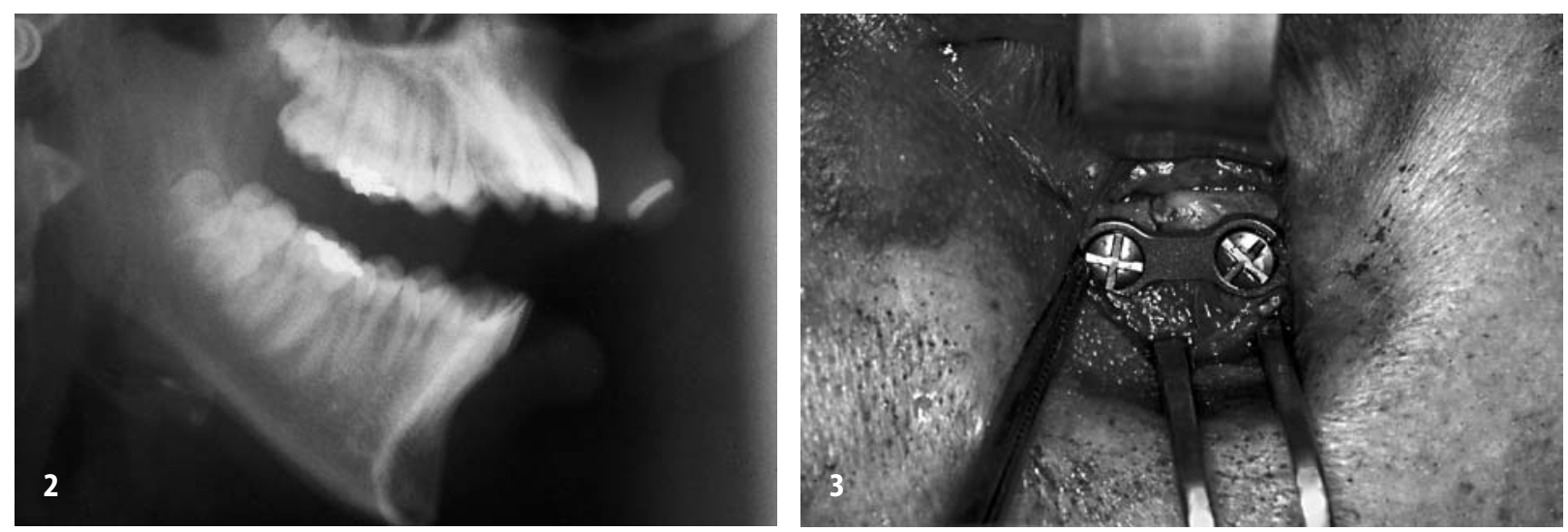

Abb. $2 \Delta$ Seitliche Schädelröntgenaufnahme: in die Oberlippe luxiertes Schmelzfragment aus einem Oberkieferfrontzahn nach einer Sturzverletzung

Abb. $3 \Delta$ Extraoraler, infraorbitaler Zugang zur Versorgung einer lateralen Mittelgesichtsfraktur. Eine der ersten multidirektionalen winkelstabilen Frakturversorgungen mit einem neu entwickelten Osteosynthesesystem 


\section{Fehlheilungen}
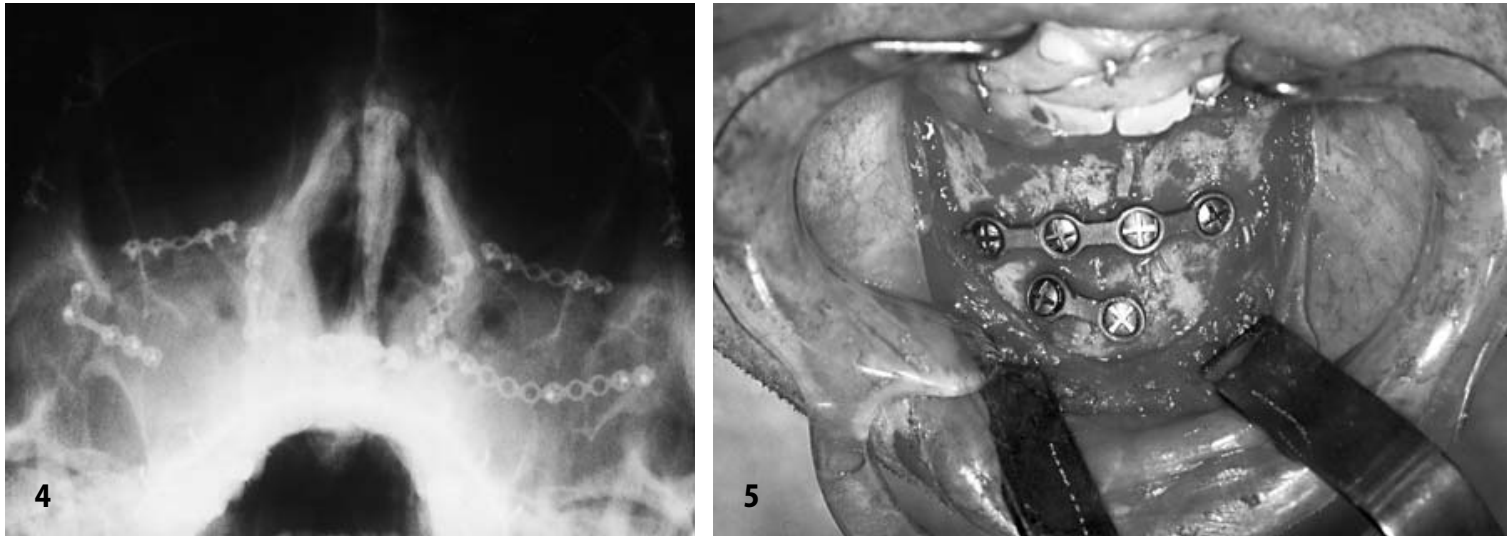

Abb. $4 \Delta$ Postoperative Röntgenkontrolle nach intra-/extraoraler Versorgung einer LeFort-III-Fraktur

Abb. $5 \Delta$ Operationssitus einer intraoralen Versorgung einer doppelten Unterkieferfraktur mit multidirektionaler winkelstabiler Osteosynthese. Am oberen Bildrand ist ein Teil einer Drahtbogenkunststoffschiene nach Schuchardt zu erkennen
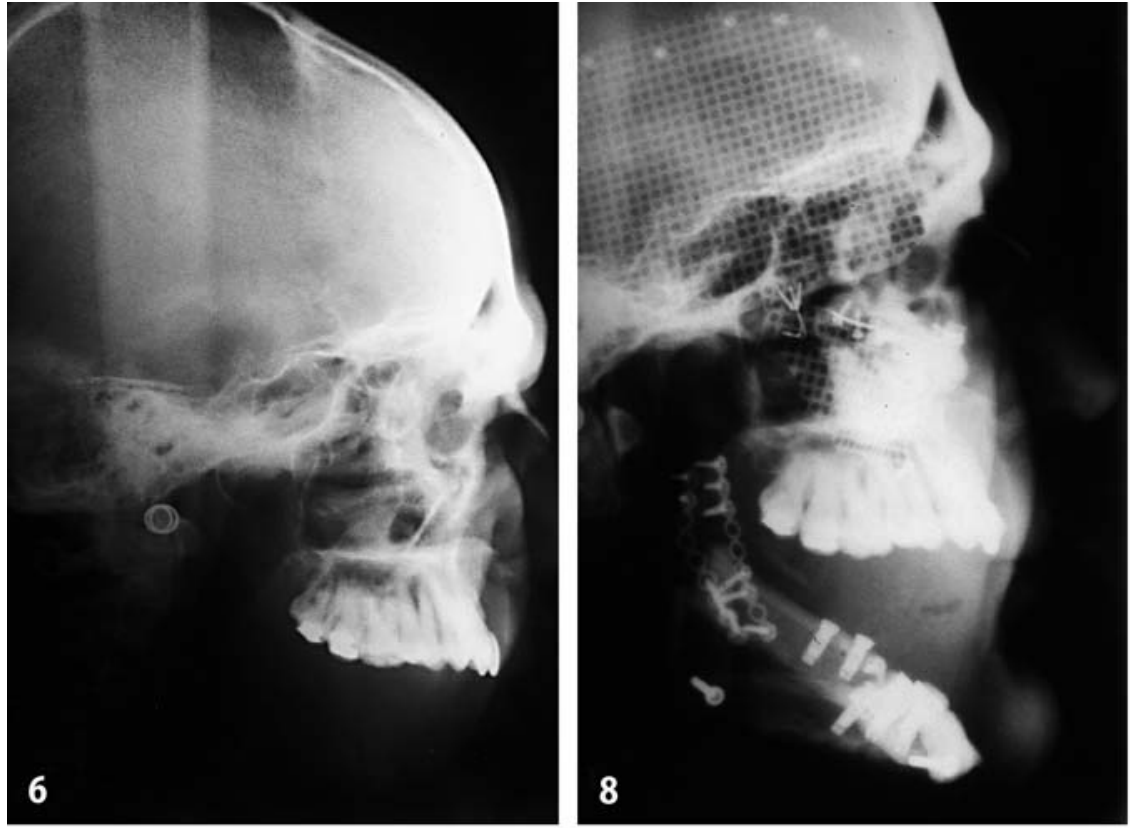

Abb. $6<$ Seitliches Röntgenbild eines Patienten nach tumorbedingter Resektion des Unterkiefers

Abb. $7 \nabla$ Mikrovaskuläres Fibulatransplantat vor Implantation und Rekonstruktion des Patienten von Abb. 6. Rechtsseitig liegt der Gefäßstumpf, das Transplantat wurde vor der Implantation bereits der ehemaligen Kieferform angepasst. Die Sicherung der Knochenfragmente erfolgt mit Miniplatten und einer Zugschraube

Abb. $8<$ Seitliches Röntgenbild nach Rekonstruktion des Unterkiefers mit einem mikrovaskulärem Fibulatransplantat. Sicherung durch Miniplattenosteosynthese. Deutlich ist die Wiederherstellung der Weichteilkontur zu erkennen

Abb. $9 \nabla$ Postoperatives Panoramaschichtröntgen des Rekonstruktionsergebnisses mit einem mikrovaskulären Fibulatransplantat nach einer Unterkieferteilresektion. Die Osteosynthese erfolgte mit Miniplatten
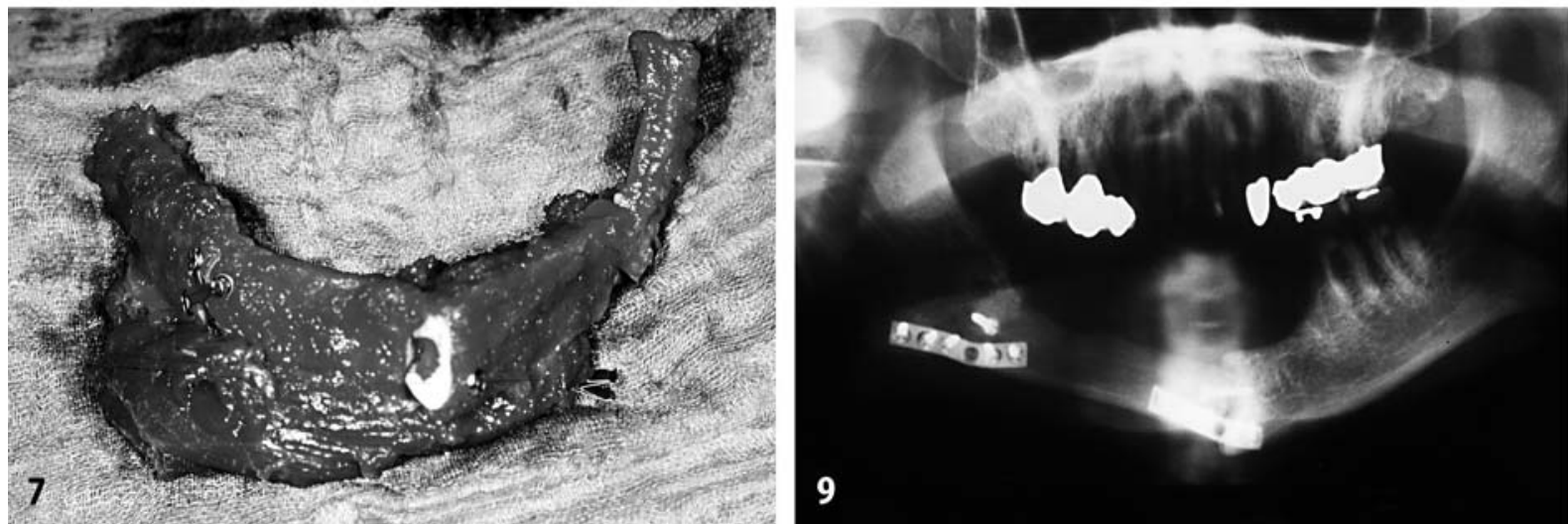
verläufe. Der basalen Absprengung der Maxilla (LeFort-I-Fraktur) folgt die pyramidale, zentrale Aussprengung des Mittelgesichts (LeFort-II-Fraktur) und, als schwerste Form der knöchernen Verletzung, die LeFort-III-Fraktur mit zentrolateraler Abtrennung des gesamten Mittelgesichts (Abb. 4). Auch kombinierte Frakturen kommen vor, der Frakturlinienverlauf der Mittelgesichtsknochen ist aber nicht immer einfach $\mathrm{zu}$ schematisieren, so ist auch eine einfachere Einteilung in zentrale und laterale Mittelgesichtsfrakturen möglich. Auch die von Wassmund modifizierte Einteilung wird der modernen Traumatologie gerecht.

\section{Frakturlokalisation}

Von den 397 Frakturen des Jahres 2000 entfielen 206 auf das Mittelgesicht und die Stirnhöhle; 169 davon betrafen das laterale Mittelgesicht, also das Jochbein und den Orbitaboden, 25 waren komplexe Mittelgesichtsfrakturen und 12 Stirnhöhlenfrakturen.

\section{Diskussion}

Zur präoperativen Diagnostik gehört neben den bekannten konventionellen Röntgenaufnahmen eine Computertomographie des Gesichtsschädels. Diese Technik ermöglicht eine bessere Beurteilung dislozierter Knochenfragmente. Auch ein Navigationssystem befindet sich in unserer Klinik im Einsatz.

Die operative Versorgung erfolgt in einem kombinierten intra- und extraoralen Eingriff von kranial-lateral nach kaudal-zentral. Eine besondere Aufmerksamkeit ist der Wiederherstellung und Sicherung und der Okklusion zu geben, sie wird mit einer Drahtbogenkunststoffschiene z. B. nach Schuchardt und Pfeiffer ermöglicht.

Schwierigkeiten beim Zusammenbiss werden von den Patienten als sehr unangenehm empfunden. Auch im höheren Alter besteht bei Indikationsstellung die Möglichkeit einer kieferorthopädischen Korrektur. Dauerhafte Sensibilitätsstörungen des Gesichts und der Zähne durch Schädigung des N. infraorbitalis treten bei bis zu 15\% der Fälle auf [5].

Eine Erfolg versprechende Innovation bei der Versorgung von Gesichts- schädelfrakturen zeichnet sich durch den Einsatz einer winkelstabilen Osteosynthese $a b[2,9,10,11]$. Aus unserer Sicht ergibt sich mit diesem System die Möglichkeit der Reduktion des Osteosynthesematerials durch eine Verringerung der Schrauben und Lochzahl bei mindestens gleicher Stabilität.

Auf den Unterkiefer entfielen 191 der 397 Frakturen des Gesichtsschädels. Die wichtige präoperative zweidimensionale Darstellung erfolgte mit einer Panoramaschichtaufnahme und einem Schädelröntgen in p.-a.-15 ${ }^{\circ}$-Richtung. Eine Computertomographie ist nur nach spezieller Indikationsstellung notwendig. Der Okklusionsrekonstruktion folgt die Frakturversorgung, im Regelfall durch eine Miniplattenosteosynthese mit einem intraoralen Zugang (Abb. 5). Ausnahmen bestehen $u$. a. bei Trümmer- oder Defektfrakturen sowie zahnlosem und extrem atrophiertem Kiefer. Hier werden Rekonstruktionsplatten mit einem extraoralen Zugang eingesetzt.

\section{Rekonstruktion}

Die Möglichkeiten der Rekonstruktion bei Gesichtsdefekten wurden durch die Weiterentwicklung und den Einsatz der Mikrochirurgie in der Mund-, Kieferund Gesichtschirurgie erheblich erweitert und verbessert. Diese moderne Technik hat aber den Einsatz der lokalen Verschiebelappen nicht verdrängt. In Abhängigkeit von der Lokalisation und der Größe des Defekts finden gestielte oder mikrochirurgisch anastomosierte Transplantate bei der intra- und extraoralen Defektdeckung Einsatz. Zur Rekonstruktion nach Tumoroperationen und Bestrahlung ermöglichen diese Transplantate eine Aufwertung der Region mit biologisch hochwertigem Gewebe. So kann die für Patienten nach Strahlentherapie unangenehme Xerostomie mit einem Dünndarmtransplantat gelindert werden.

Der Wiederaufbau von Knochendefekten erfolgt durch den Einsatz von Beckenkamm-, Skapula- oder Fibulatransplantaten (Abb. 6,7, 8,9). Bei komplexen kombinierten Knochen- und Weichteilschäden ermöglichen osteomyokutane Transplantate eine gleichzeitige Rekonstruktion von Hart- und Weichgewebe.

\section{Hinweise für die Praxis}

Gesichtsverletzte Patienten bedürfen einer exakten Untersuchung und entsprechender Diagnostik. Eine intraorale Inspektion der Zähne deckt Zahnfrakturen auf. Schmelzfragmente können in die perioralen Weichteile disloziert sein und müssen entfernt werden. Die Patienten sollten in einer Fachklinik vorgestellt werden. Patienten mit Gesichtsschädelfrakturen bedürfen einer antibiotischen Abdeckung (z. B. Clindamycin 3-mal $300 \mathrm{mg}$ peroral), da diese Frakturen primär als offen einzustufen sind. Bei Mittelgesichtsfrakturen sollten zusätzlich 5- bis 6-mal täglich Nasentropfen gegeben werden. Eine Vorstellung bei einem Ophthalmologen sollte immer erwogen werden.

\section{Literatur}

1. Andreasen J, Daugaard-Jensen J (1992) Treatment of traumatic dental injuries in children. Curr Opin Dent 15:535-550

2. Glendor U, Halling A, Andersson L (1998) Type and estimation of time spent on dental traume - a longitudinal and retrospective study. Swed Dent J 20: 15-28

3. Jackson T, Adham N (1986) Metallic plate stabilisation of bone grafts in craniofacial surgery. Br J Plast Surg 39:341-344

4. Joos U, Piffko J, Meyer U (2001) Behandlung von frontobasalen Traumen und Polytraumen. Mund Kiefer Gesichtschir 5:86-93

5. Köllner K (1995) Jochbeinfrakturen und ihre Spätfolgen, unter besonderer Berücksichtigung der Sensibilitätsstörungen und der posttraumatischen Kieferhöhlenveränderugen. Med. Dissertation, Universität Hamburg

6. Luhr H (1968) Stable osteosynthesis in fractures of the lower jaw. Dtsch Zahnärzt। Z 23: 754-60

7. Schneider E, Wimmer M, Nassut R (1996) Das Abriebverhalten von Protheseoberflächen im Knochenlager. Tribol Schmierungstechn 5:2123

8. Seebeck J, Lill C, Morlock M, Schneider E (1999) Implantatverankerung in osteoporotischen Knochen mittels monokortikaler Schrauben. Trauma Berufskrankh 1:411-414

9. Seide K, Morlock M, Schümann U, Wolter D (1999) Wirkprinzipien der winkelstabilen Platten-Schrauben-Verbindung bei Fixateurinterne-0steosynthesen. Trauma Berufskrankh 1:320-325

10. Wolter D (1993) Patentschrift Bundesrepublik Deutschland. DE 4343117 C2, 17.12.1993, AZ 4343117.8-35

11. Wolter D (1999) Fixateur-interne-Systeme. Trauma Berufskrankh 1:305-306 